\title{
Determination of Ionization and Tunneling Times in High-Order Harmonic Generation
}

\author{
Jing Zhao ${ }^{1,2}$ and Manfred Lein ${ }^{1}$ \\ ${ }^{1}$ Institut für Theoretische Physik and Centre for Quantum Engineering and Space-Time Research (QUEST), \\ Leibniz Universität Hannover, Appelstraße 2, D-30167 Hannover, Germany \\ ${ }^{2}$ Department of Physics, National University of Defense Technology, Changsha 410073, China
}

(Received 29 October 2012; published 26 July 2013)

\begin{abstract}
From the numerical solution of the time-dependent Schrödinger equation, we obtain the times of ionization and return of the laser-driven electron in high-order harmonic generation by probing the dynamics with a second harmonic field polarized orthogonal to the fundamental field and observing the harmonic emission in dependence on the two-color delay. Our retrieval method using complex-time evolution gives ionization and return times in excellent agreement with the quantum-orbit model, while a retrieval based on real-time classical dynamics can introduce substantial errors. Because of the imaginary parts, the harmonic signal polarized along the probe field is nonzero for any two-color delay. The tunneling time can be retrieved under an assumption for the return time.
\end{abstract}

High-order harmonic generation (HHG) from atoms or molecules exposed to strong laser pulses has been intensively investigated in the past two decades [1,2]. HHG as a highly nonlinear process provides a unique source of coherent extreme ultraviolet radiation in the form of single attosecond pulses or attosecond pulse trains, which paves the way for monitoring and controlling electronic dynamics on the attosecond time scale [3,4]. Because the HHG signal contains information about electronic dynamics and molecular structure, it is also extensively used for highharmonic spectroscopy [5-10].

The key to understanding HHG is the three-step model [2]: An electron tunnels into the continuum through the potential barrier formed by the atomic potential and the laser field. Then the electron is accelerated as a free particle in the strong oscillating field. Finally, the electron may recollide with the parent ion and recombine to the initial state by emission of an extreme ultraviolet photon. The photon carries away the sum of the binding energy and the electronic kinetic energy acquired in the continuum. Classical electron dynamics in the continuum is sufficient to explain approximately the cutoff in the harmonic spectrum, but the initial tunnel ionization is a quantummechanical process that requires separate treatment. A complete quantum-mechanical description, based on the strong-field approximation, was developed to give a quantitative treatment of HHG [1]. Resulting from this approach is the quantum-orbit (QO) model, where each harmonic emission frequency is attributed to a few dominant quantum trajectories evolving in complex time [11]. The question arises how accurately reality follows these model trajectories, especially since time-resolved highharmonic spectroscopy is based on the knowledge of the electron excursion times. Another question concerns the physical meaning of the imaginary parts.

With the development of time-resolved methods operating on the attosecond scale, the precise timing of electron release from an atom has been investigated for various types of ionization processes. As for single-photon ionization, isolated attosecond pulses were applied to set electrons free from the $2 s$ and $2 p$ orbitals of neon [3]. Streaking by a weak infrared field produced two different streaking traces in the photoelectron spectra. Their relative phase lag indicated an emission time difference of about 20 as. Similarly, using attosecond pulse trains, it was found that the $3 p$ photoelectrons from argon appear to be emitted about 20 as after the $3 s$ photoelectrons [4]. An entirely different ionization mechanism is laser-induced tunneling. A tunneling time characterizing the under-barrier electron motion was proposed by Keldysh in 1965 [12], but it does not seem to correspond to a measurable real delay. The arrival of the angular streaking technique [13], which provides attosecond resolution without attosecond pulses, gave experimental insight into this ionization process. The technique uses elliptically polarized laser pulses both to ionize the atom and to rotate the emission direction of the ion. The instant of ionization is thus mapped on the position of the peak in the final ion angular distribution. Very small delays between the maximum of the electric field and the maximal electron emission were found, thus supporting the concept of instantaneous tunneling [14]. Here, "instantaneous" means there is no real time delay needed to pass through the barrier. This delay must be distinguished from the Keldysh time, which is typically a few hundred attoseconds and which is almost equal to the imaginary part of the complex ionization time obtained in the QO model [15]. The angular streaking experiment measures the real delay for those electrons that tunnel at the maximum of the electric field. However, ionization can take place at any instant during the applied pulse. In fact, each harmonic order in HHG has its own ionization time (or set of times).

From the viewpoint of electron control, orthogonally polarized two-color laser pulses were proposed to monitor and control the tunneling electron wave packet [16] and to 
image molecular orbitals with attosecond resolution [17]. Recently, two-color high-harmonic spectroscopy has revealed the precise exit times of electrons in HHG [18]. The principle of the experiment is as follows. A moderate second harmonic field, polarized perpendicular to the main field, is applied in order to perturb the motion of the electron laterally once it is released from the atom. The electron trajectory depends on the phase difference between the two fields (the two-color delay). HHG is possible only when the electron returns to its initial position. Hence, the two-color delay determines the required initial lateral velocity and thus affects the harmonic intensity. The variations of harmonic intensity and recollision angle as a function of the two-color delay constitute two separate observables, facilitating the reconstruction of two quantities: ionization and return times. The experiment [18] has shown that the times at which electrons exit from the atom are well reproduced by the real parts of the ionization times from the QO model [11] and they are substantially different from the classical trajectory model [2]. In the experiment, however, there are two uncertainties. First, applying the QO model requires knowledge of the laser intensity, which is difficult to measure accurately. Second, the absolute value of the two-color delay was not measured in the experiment. Instead, the absolute scale was determined by requiring minimal averaged deviation of the retrieved return times from the $\mathrm{QO}$ return times. To overcome these limitations, we follow a theoretical approach based on the numerical solution of the time-dependent Schrödinger equation (TDSE). Although the ionization time has been investigated previously on the basis of numerical wave functions [19], it has remained an open problem to extract the dependence of the ionization time on harmonic frequency numerically, especially when attosecond resolution is desired. In this work, we follow precisely the experimental procedure, but without uncertainty about laser intensity or two-color delay. Moreover, the numerical treatment provides two independent ways to determine the return times: either from the dependence of the harmonic emission on the two-color delay as in the experiment or alternatively by inspection of the Gabor time-frequency analysis of harmonic radiation, since the electron return times coincide with the harmonic emission times. This is so unless the emission time is shifted due to a nonconstant recombination phase [20]. One challenge in the numerical approach is that the single-atom HHG spectra cannot be used directly for the two-color delay scan because every harmonic order has contributions from more than one trajectory [1]. To separate the trajectories, we use a time-frequency analysis. We find that the ionization and return times retrieved from the two-color scan deviate significantly from the QO model when we use the classical retrieval method proposed in [18]. We introduce an improved quantum-mechanical retrieval method based on trajectories evolving in complex time. Complex treatment of the lateral motion in two-color HHG has not been considered before; it explains the elliptically polarized bursts found in our TDSE results. As an alternative application of the two-color scheme, we show below that the tunneling time, which we define as the imaginary part of the complex ionization time, can be retrieved when an assumption is made about the return time.

Within the single-active-electron approximation, the TDSE for the wave function $\Psi(\mathbf{r}, t)$ describing a model $\mathrm{He}$ atom in the length gauge reads (atomic units are used throughout)

$$
i \partial_{t} \Psi(\mathbf{r}, t)=\left[\mathbf{p}^{2} / 2+V(\mathbf{r})+\mathbf{r} \cdot \mathbf{E}(t)\right] \Psi(\mathbf{r}, t)
$$

with a two-dimensional (2D) potential $V(\mathbf{r})=-1 / \sqrt{\mathbf{r}^{2}+\epsilon}$ or a three-dimensional (3D) potential $V(\mathbf{r})=-(1+$ $\left.e^{-\beta r}\right) / \sqrt{\mathbf{r}^{2}+\alpha}$, where $\mathbf{r}$ is the electron position. The softcore parameters $\epsilon, \alpha$, and $\beta$ are adjusted to reproduce the ionization potential of $\mathrm{He}\left(I_{p}=24.6 \mathrm{eV}\right)$. The electric field $\mathbf{E}(t)$ is composed of a fundamental pulse linearly polarized along the $x$ axis and a second harmonic field polarized along the $y$ axis with relative amplitude $\varepsilon=0.1$,

$$
\mathbf{E}(t)=E_{0} f(t)\left[\mathbf{e}_{x} \cos (\omega t)+\mathbf{e}_{y} \varepsilon \cos (2 \omega t+\phi)\right],
$$

where $\phi$ is the two-color delay. $E_{0}$ and $\omega$ are the amplitude and frequency of the main field with wavelength $799 \mathrm{~nm}$. For the trapezoidal profile $f(t)$, we use a total duration of three optical cycles and one-cycle linear ramps. The TDSE is solved using the split-operator method [21] with 2048 time steps per optical cycle, starting from the ground state as obtained by imaginary-time propagation. From the wave function, the dipole acceleration $\mathbf{a}(t)$ is calculated. The Gabor time-frequency analysis as a function of harmonic frequency $\Omega$ and emission time $t$ is obtained separately for the $x$ and $y$ components, i.e.,

$$
I_{\mathrm{G} x}(\Omega, t)=\left|\int d t^{\prime} a_{x}\left(t^{\prime}\right) e^{-\left(t-t^{\prime}\right)^{2} /\left(2 \sigma^{2}\right)+i \Omega t^{\prime}}\right|^{2},
$$

and similarly for $I_{\mathrm{G} y}(\Omega, t)$. We choose $\sigma=1 /(3 \omega)$ [22]. The total harmonic intensity is $I_{\mathrm{G}}(\Omega, t)=I_{\mathrm{G} x}(\Omega, t)+$ $I_{\mathrm{G} y}(\Omega, t)$. For given harmonic frequency, we obtain the emission time $t_{r}$ numerically by finding the local maximum of the Gabor intensity as a function of time. The well-known short and long trajectories [1] can be separated. Near the cutoff, the short and the long trajectories merge together. We therefore fit the numerical Gabor intensity for each harmonic order to a coherent sum of two Gaussians to determine the emission times. We focus on the short trajectory, corresponding to electrons with excursion time shorter than 0.65 optical cycles. We perform the analysis on trajectories that are born in the first half-cycle of the central part of the trapezoidal pulse.

To retrieve two quantities, namely, ionization time and return time, from the HHG spectra, we require two 
observables per harmonic order. We consider the harmonic intensity, obtained as $I_{\mathrm{G}}\left(\Omega, t_{r}\right)$ and the amplitude ratio of the $y$ and $x$ component of the generated field,

$$
R(\Omega)=\sqrt{I_{\mathrm{G} y}\left(\Omega, t_{r}\right) / I_{\mathrm{G} x}\left(\Omega, t_{r}\right)}
$$

The ratio $R$ is precisely the quantity measured in the experiment [18] where the square root of the ratio of neighboring even and odd harmonics was taken [23]. For linearly polarized harmonics, the polarization angle $\theta$ relative to the $x$ axis is given by $\tan \theta=R$. Note that our method works even when the harmonics are not linearly polarized.

The harmonic intensity and the amplitude ratio $R$ as a function of harmonic order and two-color delay are shown in Fig. 1. These results are in good agreement with the experiment. It is apparent that both the harmonic intensity and the amplitude ratio are modulated as the two-color delay is varied. The details of the modulation depend on the harmonic order. These dependencies lead to an approach to measure the electron exit times, based on the classical analysis of the electronic lateral motion after tunneling [18]. Because the second harmonic field is weak, the times of ionization $\tau_{i}$ and return $\tau_{r}$ are determined by the fundamental field. Recombination is possible if the electron returns at time $\tau_{r}$ to the position where it was released at time $\tau_{i}$, i.e., $y\left(\tau_{r}\right)-y\left(\tau_{i}\right)=0$. To satisfy this condition, the required initial velocity in the $y$ direction is

$$
v_{y 0}=-\frac{\varepsilon E_{0}}{2 \omega}\left[\sin \varphi_{i}+\frac{\cos \varphi_{r}-\cos \varphi_{i}}{2 \omega\left(\tau_{r}-\tau_{i}\right)}\right]
$$

with $\varphi_{i}=2 \omega \tau_{i}+\phi$ and $\varphi_{r}=2 \omega \tau_{r}+\phi$. The HHG efficiency is maximized for vanishing $v_{y 0}$ [24,25]. If the bound state has spherical symmetry, the amplitude ratio $R$ is fully determined by the return velocity vector, namely, $R=\left|v_{y}\left(\tau_{r}\right) / v_{x}\left(\tau_{r}\right)\right|$. In the case of linear polarization, this would mean that the recollision angle is identical to the harmonic polarization angle. We have
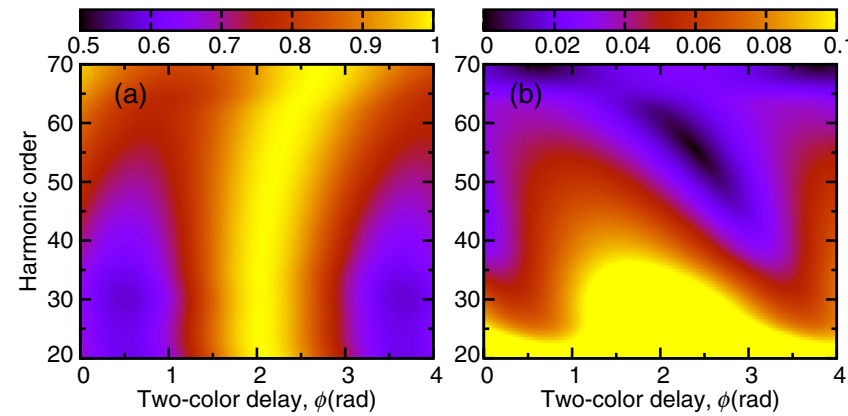

FIG. 1 (color online). (a) Normalized harmonic intensity and (b) amplitude ratio $R$ for two-color HHG as a function of harmonic order and two-color delay $\phi$, obtained from the 2D TDSE. The laser intensity is $4 \times 10^{14} \mathrm{~W} / \mathrm{cm}^{2}$. The classical cutoff is at harmonic order 65 .

$$
R=\frac{\varepsilon E_{0} /(2 \omega)}{\sqrt{2\left(\Omega-I_{p}\right)}}\left|\sin \varphi_{r}+\frac{\cos \varphi_{r}-\cos \varphi_{i}}{2 \omega\left(\tau_{r}-\tau_{i}\right)}\right| .
$$

The variations of harmonic intensity and amplitude ratio with two-color delay have been termed displacement gate and velocity gate, respectively, in [18]. From the two-color delay $\phi_{h}(\Omega)$ maximizing the harmonic intensity and the two-color delay $\phi_{a}(\Omega)$ maximizing the amplitude ratio, the ionization and return times can be retrieved. However, the classical analysis of the dynamics along the $y$ axis may not be sufficient for an accurate retrieval. A quantummechanical version has been derived for the displacement gate [18] but not for the velocity gate. Here we propose to use complex times in Eqs. (5) and (6), which remain otherwise unchanged. Using quantum orbits with complex times is the essence of our quantum-mechanical retrieval method, which is not only different from the classical and quantum retrieval methods of [18] but also conceptually simpler than the displacement-gate quantum corrections of [18]. The velocity in the $y$ direction becomes complex and the two equations determining the two-color delays $\phi_{h}$ and $\phi_{a} \mathrm{read}$

$$
\operatorname{Re} v_{y 0}=0, \quad \partial R / \partial \phi=0 .
$$

In the QO model, the ionization and return times are found by solving simultaneously the two equations $[1,11]$

$$
\begin{gathered}
{\left[p_{s}\left(\tau_{i}, \tau_{r}\right)+A\left(\tau_{i}\right)\right]^{2} / 2=-I_{p},} \\
{\left[p_{s}\left(\tau_{i}, \tau_{r}\right)+A\left(\tau_{r}\right)\right]^{2} / 2=\Omega-I_{p},}
\end{gathered}
$$

where $p_{s}\left(\tau_{i}, \tau_{r}\right)=-1 /\left(\tau_{r}-\tau_{i}\right) \int_{\tau_{i}}^{\tau_{r}} A\left(t^{\prime}\right) d t^{\prime}$ is the saddlepoint momentum and $A(t)=-\int t^{t} E\left(t^{\prime}\right) d t^{\prime}$. The solutions are the complex times $\tau_{i}=t_{i}+i \operatorname{Im} \tau_{i}$ and $\tau_{r}=$ $t_{r}+i \operatorname{Im} \tau_{r}$. The real parts $t_{i}$ and $t_{r}$ are regarded as the physical exit and return times.

As the first step in the analysis of the TDSE results, we extract for every harmonic frequency the two-color delays $\phi_{h}$ and $\phi_{a}$. For comparison, we insert the ionization and return times from the classical three-step model or the QO model into Eqs. (5) and (6) to find $\phi_{h}$ that gives $\operatorname{Re}\left(v_{y 0}\right)=0$ and $\phi_{a}$ that maximizes $R$. Figure 2 shows the results for two different laser intensities, including 3D TDSE results for the lower intensity. The comparison clearly shows that the 2D and 3D simulations give similar results. For the lower intensity, neither of the two trajectory models matches the TDSE results when the real times are used. If we use the complex times from the QO model, however, we find a very good match with the TDSE, although the QO model lacks Coulomb effects, which can be important in other strong-field situations [26]. For the higher intensity, the deficiencies of the real-time approach are less pronounced but still visible.

In the insets of Fig. 2 we show the amplitude ratio $R$ as a function of the two-color delay obtained from the TDSE, 


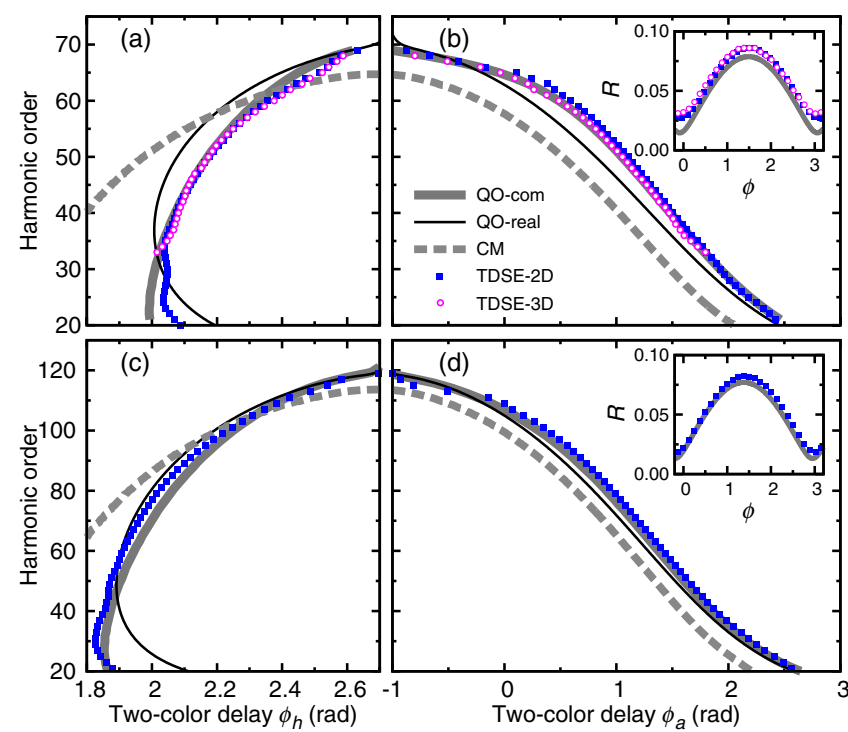

FIG. 2 (color online). Two-color delays $\phi_{h}(\Omega)$ (left) and $\phi_{a}(\Omega)$ (right). Thick solid lines (grey), QO model with complex times (QO-com). Thin solid lines (black), results using only the real parts of the QO times (QO-real). Thick dashed lines (grey), classical three-step model (CM). Purple circles and blue squares, results from the $3 \mathrm{D}$ and $2 \mathrm{D}$ TDSE, respectively. (a),(b) Intensity $4 \times 10^{14} \mathrm{~W} / \mathrm{cm}^{2}$; (c),(d) $8 \times 10^{14} \mathrm{~W} / \mathrm{cm}^{2}$. The insets show the amplitude ratio versus two-color delay for harmonic order 40 (b) and $60(d)$.

compared to Eq. (6) with the complex times from the QO model. The good agreement is in accordance with the previous observation [27] that the recollision angle in elliptically polarized fields is not strongly modified by Coulomb focusing. Interestingly the signal polarized along the $y$ axis does not become zero although classically the recollision angle must pass through zero for an appropriate choice of two-color delay. This indicates that a single recollision in the two-color field generates elliptically polarized harmonics, mathematically arising from complex return velocity components.

A problem arises in the retrieval of both complex times $\tau_{i}$ and $\tau_{r}$ from the measured two-color delays $\phi_{h}$ and $\phi_{a}$ using Eq. (7): if the times are complex, there are four unknown variables but only two equations. It is a good approximation [15] to neglect the imaginary part of $\tau_{r}$ and to assume that the tunneling time (the imaginary part of $\tau_{i}$ ) equals the Keldysh time evaluated at the instantaneous field $E_{x}\left(t_{i}\right)$, i.e., $\operatorname{Im} \tau_{i}=\sqrt{2 I_{p}} /\left|E_{x}\left(t_{i}\right)\right|$. The retrieved real parts of the ionization and return times are shown in Fig. 3(a). They match very well with the QO model. The retrieval based on classical dynamics (using real times) yields return times up to about 50 as too early; the ionization times are too early for the higher harmonic orders and too late for the low orders. Figures 3(c) and 3(d) show the deviations of the retrieved ionization and return times from the QO model. From harmonic order 40 to 55, where a clean retrieval is possible since the short-trajectory branch is perfectly isolated from
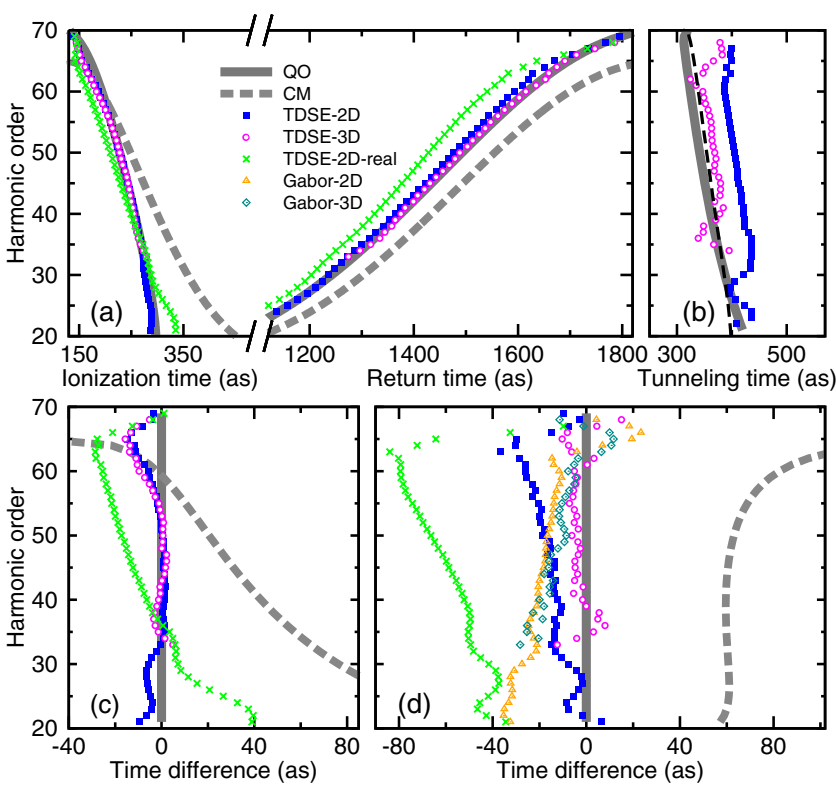

FIG. 3 (color online). (a) Reconstructed ionization times and return times. (b) Reconstructed tunneling times. Time zero in (a) is at the maximum of the electric field. Purple circles (TDSE-3D) and blue squares (TDSE-2D) represent the times from the complex-time retrieval. Green crosses represent the times from the classical retrieval with real times (TDSE-2Dreal). Thick grey solid lines show the real parts of the QO model times; thick grey dashed lines are from the classical model (CM). The thin black dashed line in (b) is the Keldysh time $\sqrt{2 I_{p}} /\left|E_{x}\left(t_{i}\right)\right|$. Bottom: ionization times (c) and return times (d) relative to the QO model. Orange triangles and cyan diamonds in $(\mathrm{d})$ are the Gabor emission times $(2 \mathrm{D}$ and $3 \mathrm{D}$, respectively). The laser intensity is $4 \times 10^{14} \mathrm{~W} / \mathrm{cm}^{2}$.

other trajectories, we find remarkably small deviations below 5 attoseconds for the $3 \mathrm{D}$ results. The error of the classical retrieval did not become apparent in the experiment [18] because the absolute scale of the two-color delay was fixed by requiring agreement with the QO model.

In an alternative scheme, we use the same observables to retrieve the real and imaginary part of the ionization time, by making suitable assumptions about the return time. We neglect the imaginary part of the return time, and we take the real part from the QO model. Note that this approach is very suitable for experiment; for example, the return times could be taken from measurements as in [11]. The retrieved imaginary part is shown in Fig. 3(b). Although the retrieval is very sensitive to small errors in the input parameters, we find good agreement with the QO model and the Keldysh time. To our knowledge, this is the only reported scheme for determination of the tunneling time.

In principle, additional observables such as the maximum and minimum of the $\phi$-dependent harmonic intensities in the $x$ and $y$ polarizations could be measured to obtain more observables for the time retrieval. Note that the imaginary parts explain why the $y$-polarized harmonic signal does not reach zero for any two-color delay (insets of Fig. 2). 
In conclusion, we have obtained the ionization and return times in high-order harmonic generation from the numerical solution of the TDSE. We use a retrieval based on complex-time trajectories and we find astonishing agreement with the QO model. The classical retrieval gives only approximate results as it ignores the change of the lateral position and velocity during tunneling. We have also retrieved the tunneling time by making reasonable assumptions for the return time. This shows the physical relevance of the tunneling time: it affects the lateral dynamics in the two-color field and makes a measurable change in the dependence on the real two-color delay. Such a position-space effect is in contrast to the view that the tunneling time determines merely the ionization rate. A signature may also be expected in the vibrational wave packet in the probing of attosecond dynamics by chirp encoded recollision [6].

We acknowledge valuable discussions with Ingo Dreißigacker.

[1] M. Lewenstein, P. Balcou, M. Y. Ivanov, A. L'Huillier, and P. B. Corkum, Phys. Rev. A 49, 2117 (1994).

[2] P. B. Corkum, Phys. Rev. Lett. 71, 1994 (1993).

[3] M. Schultze et al., Science 328, 1658 (2010).

[4] K. Klünder et al., Phys. Rev. Lett. 106, 143002 (2011).

[5] J. Itatani, J. Levesque, D. Zeidler, H. Niikura, H. Pépin, J. C. Kieffer, P. B. Corkum, and D. M. Villeneuve, Nature (London) 432, 867 (2004).

[6] S. Baker, J. S. Robinson, C. A. Haworth, H. Teng, R. A. Smith, C. C. Chirilă, M. Lein, J. W. G. Tisch, and J.P. Marangos, Science 312, 424 (2006).

[7] O. Smirnova, Y. Mairesse, S. Patchkovskii, N. Dudovich, D. Villeneuve, P. Corkum, and M. Y. Ivanov, Nature (London) 460, 972 (2009).

[8] J. Zhao and M. Lein, J. Phys. Chem. A 116, 2723 (2012).

[9] C.D. Lin, A.-T. Le, Z. Chen, T. Morishita, and R. Lucchese, J. Phys. B 43, 122001 (2010).
[10] S. Haessler, J. Caillat, and P. Salières, J. Phys. B 44, 203001 (2011).

[11] P. Salières et al., Science 292, 902 (2001).

[12] L. V. Keldysh, Sov. Phys. JETP 20, 1307 (1965).

[13] P. Eckle, M. Smolarski, P. Schlup, J. Biegert, A. Staudte, M. Schöffler, H. G. Muller, R. Dörner, and U. Keller, Nat. Phys. 4, 565 (2008).

[14] P. Eckle, A. N. Pfeiffer, C. Cirelli, A. Staudte, R. Dörner, H. G. Muller, M. Büttiker, and U. Keller, Science 322, 1525 (2008).

[15] E. V. van der Zwan and M. Lein, Phys. Rev. Lett. 108, 043004 (2012).

[16] M. Kitzler and M. Lezius, Phys. Rev. Lett. 95, 253001 (2005).

[17] M. Kitzler, X. Xie, A. Scrinzi, and A. Baltuska, Phys. Rev. A 76, 011801 (2007).

[18] D. Shafir, H. Soifer, B. D. Bruner, M. Dagan, Y. Mairesse, S. Patchkovskii, M. Y. Ivanov, O. Smirnova, and N. Dudovich, Nature (London) 485, 343 (2012).

[19] X. Xie, M. Wickenhauser, W. Boutu, H. Merdji, P. Salières, and A. Scrinzi, Phys. Rev. A 76, 023426 (2007).

[20] W. Boutu et al., Nat. Phys. 4, 545 (2008).

[21] M. D. Feit, J. A. Fleck, and A. Steiger, J. Comput. Phys. 47, 412 (1982).

[22] C. C. Chirilă, I. Dreissigacker, E. V. van der Zwan, and M. Lein, Phys. Rev. A 81, 033412 (2010).

[23] From one half cycle to the next half cycle of the fundamental field, the harmonic field along $y$ is periodic, while the harmonic field along $x$ exhibits a sign change. Thus, upon Fourier transformation to the frequency domain, the harmonics polarized along $x$ are at odd multiples of the fundamental frequency and the harmonics polarized along $y$ are at even multiples.

[24] N. Dudovich, J. Levesque, O. Smirnova, D. Zeidler, D. Comtois, M. Y. Ivanov, D. M. Villeneuve, and P. B. Corkum, Phys. Rev. Lett. 97, 253903 (2006).

[25] S. D. Khan, Y. Cheng, M. Möller, K. Zhao, B. Zhao, M. Chini, G. G. Paulus, and Z. Chang, Appl. Phys. Lett. 99, 161106 (2011).

[26] Y. Huismans et al., Science 331, 61 (2011).

[27] D. Shafir et al., Phys. Rev. Lett. 108, 203001 (2012). 